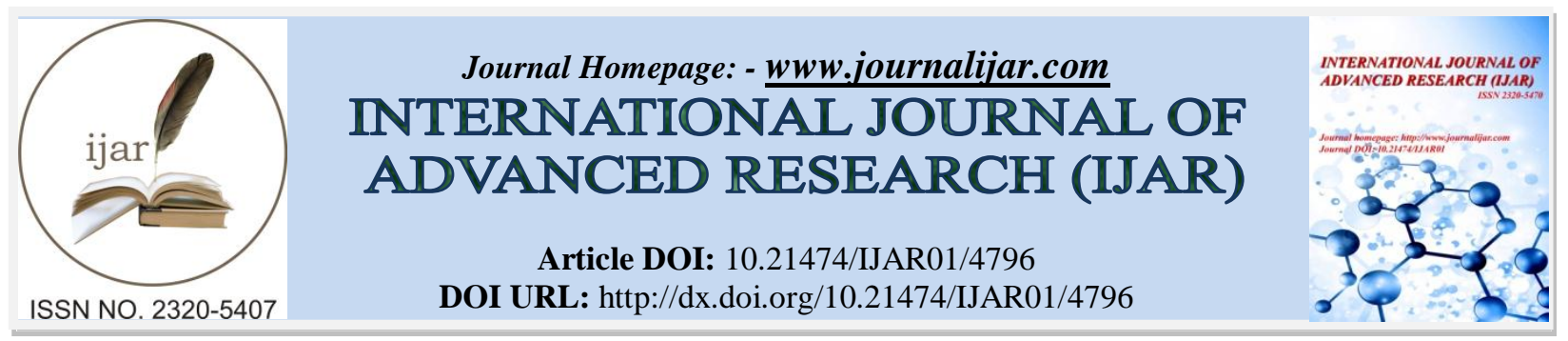

RESEARCH ARTICLE

BARYON RESONANCE SPECTRA IN DIRAC FORMALISM.

\title{
S. Panda.
}

Department of Physics, Berhampur University,Berhampur-760007,Odisha,India.

\section{Manuscript Info}

\section{Manuscript History}

Received: 9 May 2017

Final Accepted: 11 June 2017

Published: July 2017

\section{Keywords:-}

Independent quark model; chiral

symmetry; one-gluon exchange; center

of mass corrections; Gold-stone-Boson

exchange; baryon resonance spectra.
Abstract

Taking into account the color-electrostatic and magnetic energies due to one gluon exchange(OGE) interaction, along with that due to the residual quark-pion coupling arising out of the requirement of the chiral symmetry and due to necessary centre-of-mass(c.m) motion and the interactions between the constituent quarks arising out of Goldstone-Boson exchange(GBE), which are considered to play an important contributing to the energy of the Baryon core, were not taken into account in our earlier works, the baryon resonance spectra are calculated in a chiral symmetric "Potential Model of independent quarks". The effective potential representing Phenomenologically the non-perturbative gluon interactions including gluon self-couplings, is chosen with equally mixed scalar and vector parts in a linear form. The calculated values of baryon resonance spectra are found to agree reasonably well with the experiment .It is found that OGE corrections require a value of quark gluon coupling constant $\alpha_{c}=0.50$ which is consistent with the idea of treating OGE effects in low order perturbation.

Copy Right, IJAR, 2017,. All rights reserved.

\section{Introduction:-}

The present work we prefer to work based on the Dirac equation with purely phenomenological individual quark potential in an equally mixed scalar - vector potential in linear form

$$
\mathrm{V}_{\mathrm{q}}(\mathrm{r})=\frac{1}{2}\left(1+\gamma^{0}\right)\left(\mathrm{a}^{2} \mathrm{r}+\mathrm{V}_{0}\right), \mathrm{a}>0
$$

This model was found to be quite successful in describing the static electromagnetic properties, such as the magnetic moments, axial vector coupling constant ratios for beta decay processes, and the charge radii of octet baryons, as well as electromagnetic form factors of nucleons. This model is also adopted to study successfully the mass and decay constant of the ( $\mathrm{q} \bar{q}$ ) pion [1]. This model was further employed to explain reasonably well the mass spectrum of octet baryons [2], taking into account the contributions due to the color electric and magnetic energies arising out of the residual one gluon exchange (OGE) interaction, along with that due to the residual quark - pion coupling arising out of the requirement of the chiral symmetry and due to the necessary center-of-mass (c.m) motion. But in this work the interactions between the constituent quarks arising out of Goldstone -boson exchange (GBE), which are considered to play an important role in contributing to the energy of the Baryon core, were not taken into account. Therefore in the present work we intend to incorporate the GBE contributions with our model in a perturbative manner to study the mass-spectra of light and strange baryons. Here our aim is to provide a 
unified description of the ground states and the excitation spectra of baryons in the framework of this quark model, taking into account the contributions from the OGE and GBE interactions between the constituent over and above the centre-of-mass motion. We are mainly interested to see here the applicability of this model to the study of the mass spectra for some baryon resonances of the particle data group with three and four-star status [3].

In the present model, baryons are considered as systems of three constituent quarks with dynamical masses, which are confined in a first approximation by an effective linear potential and are subjected to interactions due to the OGE and GBE. For the inclusion of the GBE contributions in this model we have followed the guidelines of the chiral constituent quark models suggested by Glozman et al [4-6]. However we use these contributions in a perturbative manner along with the OGE contributions. On this context we may point that we consider the constituent quarks of flavours $\mathrm{u}, \mathrm{d}$, s with masses considerably larger than the corresponding current quark masses, so that underlying chiral symmetry of QCD is spontaneously broken (SBCS). As a consequence of SBCS at the same time Goldstone bosons appear, which couple directly to the constituent quarks $[4,6,7,8,9]$. Hence Beyond the scale of SBCS, one is left with constituent quarks with dynamical masses related to $\langle\bar{q}$ q $\rangle$ condensates and with Goldstone bosons as the effective degrees of freedom. This feature, that in the Nambu - Goldstone mode of chiral symmetry, constituent quark and Goldstone boson fields prevail together, is well supported, e.g by the $\sigma$ model [10] or the Nambu-JonaLasino (NJL) model [11]. In the same framework also the spin and flavour content of the nucleon are naturally resolved.

The work is organised in this paper as follows. In section -II we outline the potential model with the solutions for the relativistic bound states of the individually confined quarks in the $1 \mathrm{~s}, 2 \mathrm{~s}, 1 \mathrm{p}, 1 \mathrm{~d}$ and $1 \mathrm{f}$ states of baryons. Second part of the section -II provides the energy corrections due to the spurious center - of -mass motion are discussed and a brief account of the corrections due to the GBE interactions between the constituent quarks is provided in a generalized ways. This section also deals with a further correction to the baryon masses due to colour- electric and magnetic interaction energies originating from the hopefully weak residual OGE interactions, treated perturbatively. Finally, in section -III we present the results for the mass-spectra of three- four-star resonances of baryons along with those for the masses of the ground states and excited states of light-strange baryons, which are in reasonable agreement with the experimental values. This section also embodies the conclusion of the present work.

\section{Relativistic Potential Models:-}

From a phenomenological point of view we assume that the constituent quarks in a baryon core are independently confined by an average flavor - independent relativistic potential of the form given in equation (1.1). Hence, to a first approximation, the confining of the interaction represented here by an average flavor - independent potential is believed to provide zeroth order constituent quark dynamics inside such baryons. Here a and $\mathrm{V}_{0}$ are the potential parameters. We further assume that the constituent independent quarks or antiquarks obey the Dirac equation with potential $\mathrm{V}_{\mathrm{q}}(\mathrm{r})$ implying thereby a Lagrangian density of zeroth order as

$$
\mathcal{L}_{q}^{0}(\mathrm{x})=\bar{\psi}_{\mathrm{q}}(\mathrm{x})\left[\frac{i}{2} \gamma^{\mu} \overleftrightarrow{\partial}_{\mu}-\mathrm{m}_{\mathrm{q}}-\mathrm{V}_{\mathrm{q}}(\mathrm{r})\right] \Psi_{\mathrm{q}}(\mathrm{r})
$$

Which leads to Dirac equation for individual quark of mass $\mathrm{m}_{\mathrm{q}}$ as

$$
\left[\gamma^{0} \mathrm{E}_{\mathrm{q}}-\vec{\gamma} \cdot \vec{p}-\mathrm{m}_{\mathrm{q}}-\mathrm{V}_{\mathrm{q}}(\vec{r})\right] \Psi_{\mathrm{q}}(\vec{r})=0
$$

The Dirac eqn (2.2) derivable from $\mathcal{L}_{q}^{0}(x)$ can be written in two component form as

$$
\psi_{n l j}(\vec{r})=N_{n l}\left(\begin{array}{c}
\frac{i f_{n l j}(r)}{r} \\
\frac{\left(\vec{\sigma} \cdot \hat{r}_{) g_{n l j}(r)}\right.}{r}
\end{array}\right) Y_{l j m}(\mathrm{r})
$$


Here the normalized spin angular part

$$
Y_{l j m}(\mathrm{r})=\sum_{m_{l}, m_{s}}\left\langle 1, \mathrm{~m}_{\mathrm{l}}, \frac{1}{2}, \mathrm{~m}_{\mathrm{s}} \mid \mathrm{j}, \mathrm{m}_{\mathrm{j}}\right\rangle Y_{l}^{m_{l}} \chi_{\frac{1}{2}}^{m_{s}}
$$

And $N_{n l}$ is the overall normalization constant .The reduced radial part $f_{n l j}(\mathrm{r})$ of the upper component of Dirac spinor $\Psi_{\text {nlj }}$ satisfies the equation

$$
f_{n l j}^{\prime \prime}(\mathrm{r})+\left[\lambda_{\mathrm{nl}}\left\{E_{n l}^{q}-\mathrm{m}_{\mathrm{q}}-\mathrm{V}(\mathrm{r})\right\}-\frac{l(l+1)}{r^{2}}\right] f_{n l j}=0
$$

Where

$$
\lambda_{n l}=E_{n l}^{q}+\mathrm{m}_{\mathrm{q}}
$$

The present model can in principle provide the quark orbitals $\Psi_{n l j}(\vec{r})$ and the zeroth order binding energies of the confined quark for various possible eigen modes through eqns. (2.2). However, the ground state baryons, in which all the constituent quarks are in the lowest eigen states, the corresponding quark orbitals can be expressed as

$$
\Psi_{1 \mathrm{~s}}(\vec{r})=N_{n l}\left(\begin{array}{c}
\emptyset_{1 s}(\vec{r}) \\
\frac{(\vec{\sigma} \cdot \vec{p}) \emptyset_{1 s}(r)}{\lambda_{n l}}
\end{array}\right)
$$

Where $\emptyset_{1 \mathrm{~s}}(\vec{r})$ is the radial angular part of the upper component $\Psi_{1 \mathrm{~s}}(\vec{r})$ and is given by

$$
\emptyset_{1 \mathrm{~s}}(\vec{r})=\frac{i}{\sqrt{4 \pi}} \frac{f_{1 s}(r)}{r}
$$

For the ground state eqn (2.5) reduces to

$f_{1 s}^{\prime \prime}(\mathrm{r})+\left[\lambda_{1 s}\left(\mathrm{E}_{1 \mathrm{~s}}-\mathrm{m}_{\mathrm{q}}-\mathrm{a}^{2} \mathrm{r}-\mathrm{V}_{0}\right)\right] \mathrm{f}_{1 \mathrm{~s}}(\mathrm{r})=0$

which can be transformed into a convenient dimensionless form

$$
f_{1 s}^{\prime \prime}(\rho)+\left(\epsilon_{1 \mathrm{~s}}-\rho\right) \mathrm{f}_{1 \mathrm{~s}}(\rho)=0
$$

Where $\left(\rho=\frac{r}{r_{0 q}}\right)$ is a dimensionless variable with $r_{0 q}=\left(\lambda_{\mathrm{q}} \mathrm{a}^{2}\right)^{-1 / 3}$

$$
\text { and } \epsilon_{1 \mathrm{~s}}=\left(\frac{\lambda_{1 s}}{a^{4}}\right)^{1 / 3}\left[E_{1 s}^{0}-\mathrm{m}_{\mathrm{q}}-\mathrm{V}_{0}\right]
$$

The eqn (2.9) is the basic eigen value equation, which can be solved as follows: with $\mathrm{z}=\rho \in_{\mathrm{q}}, \mathrm{f}_{\mathrm{q}}=\mathrm{f}_{1 \mathrm{~s}}$ and $\epsilon_{\mathrm{q}}=\epsilon_{1 \mathrm{~s}}$ eqn.(2.9) reduces to the Airy equation

$$
f_{q}^{\prime \prime}(\mathrm{z})-\mathrm{zf}_{\mathrm{q}}(\mathrm{z})=0
$$

The solution $f_{q}(z)$ of equation (2.11) is the Airy function $A_{i}(z)$. Since at $r=0$ we require $f_{q}(r)=0$, we have $A_{i}(z)=0$ at $\mathrm{z}=-\epsilon_{\mathrm{q}}$. If $\mathrm{z}_{\mathrm{q}}$ are the roots of the Airy function such that $\mathrm{A}_{\mathrm{i}}\left(\mathrm{z}_{\mathrm{n}}\right)=0$, then we have $\mathrm{z}=-\epsilon_{\mathrm{q}}=\mathrm{z}_{\mathrm{n}}$. For the ground state of quarks or antiquarks, the $\epsilon_{1 \mathrm{~s}}$ value is given by the first root $z_{1}$ of the airy function so that

$$
\epsilon_{\mathrm{q}}=\epsilon_{1 \mathrm{~s}}=-\mathrm{z}_{1}
$$

the value of this root $\mathrm{z}_{1}=-2.33811$ 
The eigen value $\epsilon_{\mathrm{q}}$ and $\mathrm{f}_{\mathrm{q}}$ by W.K.B method which can yield a solution (2.9) as per [2] in the form

$$
\begin{gathered}
\mathrm{f}_{\mathrm{q}}(\rho)=\left[\mathrm{A}_{\mathrm{q}} /\left(\epsilon_{\mathrm{q}}-\rho\right)^{1 / 4}\right] \cos \eta_{\mathrm{q}} \\
\text { where } \eta_{\mathrm{q}}=\int_{0}^{\rho} d \rho^{\prime}\left(\epsilon_{\mathrm{q}}-\rho^{\prime}\right)^{-1 / 2}-\frac{\pi}{4} \\
\text { and } \epsilon_{\mathrm{q}}=\frac{9}{4}\left(\frac{\pi}{3}\right)^{2 / 3}
\end{gathered}
$$

and $\mathrm{A}_{\mathrm{q}}=$ normalization constant $=\left(\epsilon_{\mathrm{q}} / r_{0 q}\right)^{1 / 2}$. Now the individual quark binding energy $\mathrm{E}_{\mathrm{q}}$ of zeroth order in ground state can be obtained from eqn.(2.10) through the relations

$$
\begin{gathered}
\mathrm{E}_{\mathrm{q}}=m_{q}+\mathrm{V}_{0}+\mathrm{a} x_{q} \\
\left(\epsilon_{1 \mathrm{~s}}\right)^{3}=\frac{\lambda_{q}}{a^{4}} \mathrm{a}^{3} x_{q}^{3}=\frac{\left(E_{q}+m_{q}\right)}{a} x_{q}^{3} \\
\epsilon_{q}^{3}=\frac{\left(m_{q}+V_{0}+a x_{q}+m_{q}\right)}{a} x_{q}^{3} \\
\in_{q}^{3}=x_{q}^{4}+\frac{\left(2 m_{q}+V_{0}\right)}{a} x_{q}^{3}=x_{q}^{4}+\mathrm{b} x_{q}^{3} \\
\text { Where } \mathrm{b}=\frac{\left(2 m_{q}+V_{0}\right)}{a} \\
\mathrm{x}_{q}{ }^{4}+\mathrm{b} x_{q}^{3}-\in_{q}^{3}=0
\end{gathered}
$$

Then using $\epsilon_{\mathrm{q}}$ value from the solution of eqn.(2.9) and 'b' from (2.36) with known potential parameters $\mathrm{V}_{0}$, a and $\mathrm{m}_{\mathrm{q}}$, the eqn.(2.17) can be solved to get an unique value of $x_{q}$, which when substituted in eqn.(2.16) yield the independent quark binding energy $\mathrm{E}_{\mathrm{q}}$. The independent quark wave function $\Psi_{\mathrm{q}}(\vec{r})$ given by the eqn.(2.7) and the corresponding energy $\mathrm{E}_{\mathrm{q}}$ describe the relativistic bound states of the confined quarks of baryons.

For ground state, the quark wave function can also be written in two component form as

$$
\Psi_{\mathrm{q}}(\vec{r})=\mathrm{N}_{\mathrm{q}}\left(\begin{array}{c}
\emptyset_{q}(\vec{r}) \\
\frac{\vec{\sigma} \cdot \vec{p}}{\lambda_{q}} \emptyset_{q}(\vec{r})
\end{array}\right) \chi \uparrow
$$

Where $\lambda_{\mathrm{q}}=\mathrm{E}_{\mathrm{q}}+m_{q}$

and

$$
\emptyset_{\mathrm{q}}(\vec{r})=\frac{i f_{q}(r)}{r} y_{0}^{0}(\theta, \varnothing)
$$

$\emptyset_{\mathrm{q}}(\vec{r})$ is the normalized radial angular part of $\Psi_{\mathrm{q}}(\vec{r})$. The overall normalization constant $\mathrm{N}_{\mathrm{q}}$ of $\Psi_{\mathrm{q}}(\vec{r})$ In the equation (2.19) can be obtained in a simplified form as

$$
N_{q}^{2}=\frac{3\left(E_{q}+m_{q}\right)}{\left(4 E_{q}+2 m_{q}-V_{0}\right)}
$$

Solution for the quark binding energy $E_{1 s}^{q}$ in zeroth order corresponding to the ground state of the baryon immediately leads to the ground state mass of the baryon core in zeroth order as

$$
M_{B}^{0}=E_{B}=\sum_{q} E_{1 s}^{q}
$$

In this model eqn.(2.5) is then solved for $2 \mathrm{~s}, 1 \mathrm{p}$ states to obtain the individual quark binding $E_{2 s}^{q}, E_{1 p}^{q}$ respectively, with the help of a standard numerical method which yields $\epsilon_{2 \mathrm{~s}}=4.08741, \epsilon_{1 \mathrm{p}}=3.3611$. These values lead to the 
corresponding masses of the excited states of baryon core in zeroth order in the same way as in case of ground state. The overall normalization constant $\mathrm{N}_{\mathrm{nl}}$ of $\Psi_{\mathrm{nlj}}(\vec{r})$ appearing in eqn.(2.3) is of the form

$$
N_{n l}^{2}=\left[\left[1+\left(E_{n l}^{q}-m_{q}-\mathrm{V}_{0}-\mathrm{a}\langle r\rangle_{\mathrm{nl}}\right] / \lambda_{\mathrm{nl}}\right]^{-1}\right.
$$

where $\langle r\rangle_{\mathrm{nl}}$ is the expectation value of $\mathrm{r}$ with respect to $\emptyset_{\mathrm{nlj}}(\vec{r})$

\section{Energy correction to baryon masses:-}

The contribution of quark binding energy to the mass of the baryon core given by the eqn.(2.22) needs correction due to center -of-mass motion, Goldstone boson exchange (GBE) interactions and quark-gluon interaction which need to be calculated separately in order to obtain the physical mass of the baryons.

\section{A Centre - of-Mass Correction:-}

Due to the mass of the center-of-mass of the three quark system, there would be sizeable correction to the energy of baryons. Our shell type relativistic quark model is not transitionally invariant which means the independent motion of the quarks inside the hadron-core does not lead to a state of definite total momentum, as it should to represent the physical state of baryons. The problem appears in the same way in nuclear physics in case of $\mathrm{He}^{3}$ and also in MIT bag model [12 ] and therefore has to be resolved accordingly [13]. Here we adopt the prescriptions of Wong [14] and other workers, which is just one way of accounting for the centre-of-mass motion. In such a prescription[x], the physical mass $M_{B}^{0}$ of the baryon core is related to the relativistic energy $E_{B}$ of the three quark states as

$$
M_{B}^{0}=\left(E_{B}^{2}-<p_{B}^{2}>\right)^{1 / 2}
$$

Where $E_{B}=\sum_{q} E_{q}, \vec{p}_{B}$ is the centre - of - mass momentum and $\left\langle\vec{p}_{B}^{2}\right\rangle$ is evaluated with

usual approximation as $\quad<\vec{p}_{B}^{2}>=\sum_{q}<\vec{p}^{2}>_{\mathrm{q}}$

where $<\vec{p}_{q}^{2}>$ is the average value of the square of the individual quark momentum taken over / $s_{\frac{1}{2}}$ single -quark states and is given in this model as

$$
<\vec{p}_{q}^{2}>=\frac{\left(E_{q}+m_{q}\right)\left(E_{q}-m_{q}-V_{0}\right)\left(8 E_{q}-3 \mathrm{~V} 0+2 m_{q}-3 V_{0}\right)}{5\left(4 E_{0}-V_{0}+2 m_{q}\right)}
$$

In the same way, one can find the expression for the centre of mass corrected mass of the bare baryon core as

$$
E_{B}=\left(\left(E_{B}^{0}\right)^{2}-\sum_{q}<\vec{p}_{q}^{2}>\right)^{1 / 2}
$$

which provides the necessary c.m correction to the energy as

$$
\left(\Delta E_{B}\right)_{\mathrm{c} \cdot \mathrm{m}}=\left(E_{B}-E_{B}^{0}\right)=\left(\left(E_{B}^{0}\right)^{2}-\sum_{q}<\vec{p}_{q}^{2}>\right)^{1 / 2}-E_{B}
$$

\section{B Correction due to Goldstone Boson Exchange interaction (GBE):-}

The $\mathrm{SU}(3)_{\mathrm{L}} \otimes \mathrm{SU}(3)_{\mathrm{R}}$ chiral symmetry of QCD Lagrangian's spontaneously broken down to SU(3) by the QCD vacuum [ in large $\mathrm{N}_{\mathrm{c}}$ limit it would be $\mathrm{U}(3)_{\mathrm{L}} \otimes \mathrm{U}(3)_{\mathrm{R}} \rightarrow \mathrm{U}(3)_{\mathrm{v}}$ ]. There are two important generic consequences of SBCS. The first one is an appearance of the octet of pseudoscalar mesons of low mass $\pi, \mathrm{k}, \eta$ which represent the associated approximate Goldstone bosons (in the large $\mathrm{N}_{\mathrm{c}}$ limit the flavour singlet state $\eta$ ' should be added ). The second one is that valence (partially massless) quarks acquire a dynamical mass, which has been called historically 
constituent mass. Indeed, the nonzero value of quark condensate, $\left\langle\bar{q} q>\sim-(250 \mathrm{Mev})^{3}\right.$, itself implies at the formal level that there must be at low momenta a rather big dynamical mass, which should be a momentum dependent quantity. Such a dynamical mass is now directly observed on the lattice [15].

Thus the constituent quarks should be considered as quasi -particles whose dynamical mass at low momenta comes from the non-perturbative gluon and quark-antiquark dressing. The flavor- octet axial current conservation in the chiral limit tells that the constituent quarks and Goldstone bosons should be coupled with the strength $g=\frac{g_{A} M}{f_{\pi}},[16]$ which is a quark analog of the famous Goldberger-Treiman relation. It has been recently suggested that in the low energy regime, below the chiral symmetry breaking scale $\sim 1 \mathrm{Gev}$, the low lying light and strange baryons should be predominantly viewed as systems of three constituent quarks with an effective confining interaction mediated by GBE between the constituent quarks [17].

The coupling of Goldstone bosons ( $\pi, \eta \& \mathrm{k}$ mesons ) to the constituent quarks arising from SBCS in QCD can be taken into account in a perturbative manner in the same way as it has been done in the study of the effect of quarkpion coupling in the CBM [18].Here the fields of the Goldstone bosons may be treated independently without any constraint and their interactions with the quarks can be assumed to be linear as it is done in case of pion[19].

Following the Hamiltonian technique [20] as has been used in CBM, we can describe the effect of Goldstone bosons ( i.e meson, $\chi=\pi, \eta, \mathrm{k}$ ) in low order perturbation theory as follows. The pionic self - energy of the baryons can be evaluated with the help of the single-loop self energy diagram (fig (2.1) as

$$
\sum_{B}\left(\mathrm{E}_{\mathrm{B}}\right)=\sum_{K} \sum_{\mathrm{B}^{\prime}} \frac{V^{+\mathrm{BB}^{\prime}} V^{\mathrm{BB}}}{E_{B}-\omega_{k}-M_{\mathrm{B}^{\prime}}^{0}}
$$

where $\sum_{K}=\sum_{j} \int \frac{d^{3} \bar{k}}{(2 \pi)^{3}}$. Here $\mathrm{j}$ corresponds to the pion-isospin index and $\mathrm{B}^{\prime}$ is the intermediate baryon state. And $V^{B^{\mathrm{B}^{\prime}}}(\vec{K})$ is the general baryon-pion absorption vertex function obtained in this model as

$$
V_{j}^{B \mathrm{~B}^{\prime}}(\vec{K})=\mathrm{i}(\sqrt{ } 4 \pi) \frac{f_{B B^{\prime} \pi}}{m_{\pi}} \frac{k u(k)}{\left(\sqrt{ } 2 \omega_{k}\right)}\left(\vec{\sigma}^{B B^{\prime}} \cdot \hat{k}\right) \times \tau_{j}^{B \mathrm{~B}^{\prime}}
$$

where $\vec{\sigma}_{j}^{B \mathrm{~B}^{\prime}}$ and $\tau^{B \mathrm{~B}^{\prime}}$ are spin and isospin matrices and $\omega_{k}^{2}=\vec{k}^{2}+m_{\pi}^{2}$. The form factor $\mathrm{u}(\mathrm{k})$ in this model can be expressed as

$\left.\mathrm{u}(\mathrm{k})=\frac{5 N_{q}^{2}}{3 \lambda_{q} g_{A}}\left[\left(2 m_{q}+V_{0}\right) \ll j_{0}(|\vec{k}| \mathrm{r}) \gg+a^{2} \ll r j_{0}(|\vec{k}| \mathrm{r}) \gg+a^{2} \ll j_{1}(|\vec{k}| \mathrm{r}) / \mathrm{k}\right) \gg\right]$

Where $j_{0}(|\vec{k}| \mathrm{r})$ and $j_{1}(|\vec{k}| \mathrm{r})$ represent the zeroth and first order spherical Bessel functions, respectively.The double angular brackets stand for the expectation values with respect to $\emptyset_{m}(\mathrm{r})$. In this model the axial vector coupling constant $g_{A}$ for the beta decay of the neutron is given by

$$
g_{A}^{0}=\frac{5}{9}\left(\frac{8 E_{q}+10 m_{q}+V_{0}}{4 E_{q}+2 m_{q}-V_{0}}\right)
$$

Now, with the vertex function $V_{j}^{B \mathrm{~B}^{\prime}}(\vec{k})$ at hand, it is possible to calculate the pionic self- energy for various baryons with appropriate baryon intermediate states contributing to the process. For degenerate intermediate states on mass shell with $M_{B}^{0}=M_{\mathrm{B}^{\prime}}^{0}$ the self energy correction becomes

$$
\left(\delta M_{B}\right)_{\pi}=\sum_{B} \quad\left(E_{B}^{0}=M_{B}^{0}=M_{\mathrm{B}^{\prime}}^{0}\right)=-\sum_{K, B} \frac{V^{+B \mathrm{~B}^{\prime}} V^{B \mathrm{~B}^{\prime}}}{\omega_{k}}
$$


Now using eqn ( 91 ), we find

$$
\left(\delta M_{B}\right)_{\pi}=-\frac{I_{\pi}}{3} \sum_{B^{\prime}} C_{B B^{\prime}} f_{B B \pi}^{2}
$$

$$
\begin{array}{r}
\text { Where } C_{B B^{\prime}}=\left(\vec{\sigma}^{B B^{\prime}} \cdot \vec{\sigma}^{B^{\prime} B}\right)\left(\vec{\tau}^{B B^{\prime}} \cdot \vec{\tau}^{B^{\prime} B}\right) \\
\text { and } I_{\pi}=\frac{1}{\pi m_{\pi}^{2}} \int_{0}^{\infty} \frac{d k k^{4} u^{2}(k)}{\omega_{k}^{2}}
\end{array}
$$

For intermediate baryon states $\mathrm{B}^{\prime}$ we consider only the octet and decuplet ground states. The self-energy $\left(\delta M_{B}\right)_{\pi}$ for different baryons can be computed by using the values of $f_{B B^{\prime} \pi}$ and $C_{B B^{\prime}}$ [2].The self - energy $\left(\delta M_{B}\right)_{\pi}$ calculated here contains both the quark self energy fig(2.2a) and the one -pion exchange contributions fig (2.2b) . It must be noted here that this method ignores to a large extent the short range part of pion exchange interaction, which is of crucial - importance for splittings. Only when the complete infinite set of all radially excited intermediate state $\mathrm{B}$ 'is taken into account, the method could be adequate[21]. For example, the meson exchange contribution to the N$\Delta$ difference will become much larger. It will also be strongly enhanced when meson exchange contribution is strongly dependent on the radious of the bare wave function i.e on the type of confinement. This dependence has not been studied in the present work.

The corrections due to $\eta$ - exchange and kaon exchange interactions between the constituent quark can be calculated following same approach as we have just used in case of pion. Thus in general, one can write energy corrections due to GBE interactions as

$$
\left(\delta M_{B}\right)_{\chi}=-\frac{I_{\chi}}{3} \sum_{B^{\prime}} \mathrm{C}_{\mathrm{BB}^{\prime}} f_{B B^{\prime} \chi}^{2}
$$

Where $\mathrm{C}_{\mathrm{BB}}$ ' is given by eqn ( 2.35 )

And

$$
\mathrm{I}_{\chi}=\frac{1}{\chi m_{\chi}^{2}} \int_{0}^{\infty} \frac{d k k^{4} u^{2}(k)}{k^{2}+m_{\pi}^{2}}
$$

Following the discussions given in ref [22] the baryon-meson coupling constant $f_{N N^{\prime} \chi}$. Pion exchange interaction acts only between light quarks where as $\eta$ - exchange is allowed in all quark pair states .The kaon exchange interaction takes place in $\mathrm{u} \sim \mathrm{s}$ and $\mathrm{d} \sim \mathrm{s}$ pair states.

\section{II.2C. One Gluon Exchange Correction:-}

The individual quarks in a baryon core have been considered so far to be experiencing the only force coming from the average effective potential $V_{q}(r)$ in equation (2.1), which is assumed to provide a suitable phenomenological description of the non-perturbative gluon interaction including the gluon self-couplings. All that remains inside the quark core is the hopefully weak one-gluon-exchange interaction provided by the interaction Lagrangian density

$$
\mathcal{L}_{I}^{g}=\sum_{\alpha=1}^{\infty} J_{i}^{\mu \alpha}(\mathrm{x}) A_{\mu}^{\alpha}(x)
$$

Where $A_{\mu}^{\alpha}(x)$ are the eight - vector gluon fields and $J_{i}^{\mu \alpha}(\mathrm{x})$ is the ith quark color current. Since at small distances the quarks should be almost free, it is reasonable to calculate the energy shift in the mass spectrum arising out of the quark interaction energy due to their coupling to the colored gluons, using a first order perturbation theory. Taking into account the specific quark flavour and spin configurations in various ground state baryons and using the relations $\left\langle\sum_{\alpha}\left(\lambda_{i}^{2}\right)^{2}\right\rangle=\frac{16}{3}$ and $\left\langle\sum_{\alpha} \lambda_{i}^{\alpha} \lambda_{j}^{\alpha} \quad\right\rangle_{\mathrm{i}} \neq \mathrm{j}=-\frac{8}{3}$ for baryons, in general one can write the energy correction due to one - gluon- exchange as per our earlier work[2]. 


$$
\begin{gathered}
\left(\Delta E_{B}\right)_{g}^{e}=\alpha_{c}\left(a_{u u} T_{u u}^{e}+a_{u s} T_{u s}^{e}+a_{s s} T_{s s}^{e}\right) \\
\left(\Delta E_{B}\right)_{g}^{m}=\alpha_{c}\left(b_{u u} T_{u u}^{m}+b_{u s} T_{u s}^{m}+b_{s s} T_{s s}^{m}\right)
\end{gathered}
$$

where $\mathrm{a}_{\mathrm{ij}}$ and $\mathrm{b}_{\mathrm{ij}}$ are the numerical coefficient depending on each baryons listed in Table -2.1 and the terms $T_{i j}^{e, m}$ are

$$
\begin{aligned}
T_{i j}^{e} & =\frac{48\left(E_{i}+m_{i}\right)\left(E_{j}+m_{j}\right)}{\pi\left(4 E_{i}+2 m_{i}-V_{0}\right)\left(4 E_{j}+2 m_{j}-V_{0}\right)} \\
T_{i j}^{m} & =\frac{32\left(E_{i}+m_{i}\right)\left(E_{j}+m_{j}\right)}{\pi\left(4 E_{i}+2 m_{i}-V_{0}\right)\left(4 E_{j}+2 m_{j}-V_{0}\right)} I_{i j}^{m}
\end{aligned}
$$

From table 2.1 one can note that the color - electric contribution for the baryon masses vanishes when all the constituent quark masses in a baryon are equal, where as it is non-zero otherwise. However, even in the case of strange baryons, it would subsequently be seen that the colour - electric contribution is quite small. Therefore, the degeneracy among the baryons is essentially removed the spin - spin interaction energy in the colour magnetic part.

Table-1:-The coefficients $a_{i j}$ and $b_{i j}$ used in calculation of the color- electric and color -magnetic energy contributions due to one-gluon exchange:

\begin{tabular}{|c|c|c|c|c|c|c|}
\hline Baryons & $a_{u u}$ & $a_{u s}$ & $a_{s s}$ & $b_{u u}$ & $b_{u s}$ & $b_{s s}$ \\
\hline $\mathrm{N}$ & 0 & 0 & 0 & -3 & 0 & 0 \\
\hline$\Delta$ & 0 & 0 & 0 & 3 & 0 & 0 \\
\hline$\Lambda$ & 1 & -2 & 1 & -3 & 0 & 0 \\
\hline$\Sigma$ & 1 & -2 & 1 & 1 & -4 & 0 \\
\hline$\Sigma^{*}$ & 1 & -2 & 1 & 0 & -4 & 2 \\
\hline$\Xi$ & 1 & -2 & 1 & 1 & 2 & 0 \\
\hline$\Xi^{*}$ & 1 & -2 & 1 & 0 & 2 & 1 \\
\hline$\Omega^{-}$ & 0 & 0 & 0 & 0 & 0 & +3 \\
\hline
\end{tabular}

Table 2:-The calculated values of $T_{i j}^{e, m}$ and $I_{\chi}$, Required respectively for OGE and GBE Contributions in Mev .

\begin{tabular}{|c|c|c|c|c|c|c|c|}
\hline LS Multiplet & $\left(\delta M_{B}\right)_{\pi}$ & $\left(\delta M_{B}\right)_{K}$ & & $\left(\delta M_{B}\right)_{\eta}$ & & & $\left(\delta M_{B}\right)_{\chi}$ \\
\hline & & & $\left(\delta M_{B}\right)_{\eta}^{u u}$ & $\left(\delta M_{B}\right)_{\eta}^{u u}$ & $\left(\delta M_{B}\right)_{\eta}^{u u}$ & Total & \\
\hline $\mathrm{N}$ & -236.60 & 0 & 313.30 & 0 & 0 & 313.30 & 76.70 \\
\hline$\Delta$ & -136.98 & 0 & 284.12 & 0 & 0 & 284.12 & 147.14 \\
\hline$\Lambda$ & -149.43 & 199.46 & 14.86 & 46.92 & 0 & 61.78 & 111.81 \\
\hline$\Sigma$ & -83.02 & 188.88 & 14.86 & 46.92 & 0 & 61.78 & 167.64 \\
\hline$\Sigma^{*}$ & -83.02 & 188.88 & 14.86 & 46.92 & 0 & 61.78 & 167.64 \\
\hline$\Xi$ & -37.35 & 84.99 & 0 & 46.91 & 74.06 & 120.97 & 168.61 \\
\hline$\Xi^{*}$ & -37.35 & 84.99 & 0 & 46.91 & & 120.97 & 168.51 \\
\hline$\Omega^{-}$ & 0 & 0 & 0 & 0 & 222.18 & 222.18 & 222.18 \\
\hline$\frac{1}{2}^{-}, N(1140)$ & -23.99 & 0 & -204.37 & 0 & 0 & -204.37 & -228.30 \\
\hline$\frac{3^{-}}{2}, N(1520):$ & -68.41 & 0 & -5.08 & 0 & 0 & -5.08 & -73.49 \\
\hline
\end{tabular}

\begin{tabular}{|l|l|l|l|l|l|l|l|l|l|}
\hline & \multicolumn{3}{|c|}{$T_{i j}^{m}$} & \multicolumn{1}{c|}{$T_{i j}^{e}$} & \multicolumn{1}{c|}{$I_{\chi}$} \\
\hline & \multicolumn{1}{|c|}{$T_{u u}^{m}$} & \multicolumn{1}{|c|}{$T_{u s}^{m}$} & \multicolumn{1}{c|}{$T_{s s}^{m}$} & $T_{u u}^{e}$ & $T_{u s}^{e}$ & $T_{s s}^{e}$ & $I_{\pi}$ & $I_{\chi}$ & $I_{\eta}$ \\
\hline $1 \mathrm{~S}$ & 73.85 & 64.63 & 62.90 & 375.67 & 402.20 & 443.76 & 431.90 & 26.29 & 20.49 \\
\hline $2 \mathrm{~S}$ & 5.47 & 5.16 & 5.20 & 279.25 & 291.54 & 306.08 & 43.81 & 1.83 & 1.38 \\
\hline $1 \mathrm{P}$ & 13.72 & 12.70 & 12.55 & 328.93 & 345.20 & 364.96 & 124.88 & 6.42 & 4.90 \\
\hline $1 \mathrm{D}$ & 4.61 & 4.34 & 4.31 & 274.28 & 285.66 & 298.96 & 40.26 & 1.65 & 1.23 \\
\hline $1 \mathrm{~F}$ & 2.11 & 1.99 & 1.96 & 235.97 & 244.78 & 254.89 & 16.47 & 0.54 & 0.390 \\
\hline
\end{tabular}

Table-3:-GBE Corrections $\left(\delta M_{B}\right)_{\chi}(\chi=\pi, \eta, \mathrm{K})$ for the 1S, 2S, and 1P States of Light-and Strange-

Baryons in Mev. 


\begin{tabular}{|c|c|c|c|c|c|c|c|}
\hline$\frac{1^{-}}{2}, N(1535)$ & & & & & & & \\
\hline$\frac{3}{2}, \Delta(1600)$ & -13.89 & 0 & -70.88 & 0 & 0 & -70.88 & -84.77 \\
\hline $\begin{array}{cc}\frac{1^{-}}{2}, & \Delta \\
(1620): & \\
\frac{3}{2}^{-}, & \Delta \\
(1700) & \end{array}$ & -39.60 & 0 & 57.94 & 0 & 0 & 57.94 & 18.34 \\
\hline $\begin{array}{l}\frac{1^{-}}{2}, \quad \Lambda \\
(1405): \\
\frac{3}{2}^{-} \\
\Lambda(1520) \\
\end{array}$ & -43.20 & -216.58 & -2.19 & -6.32 & 0 & -8.51 & -268.29 \\
\hline $\begin{array}{cc}\frac{1}{2}^{-}, & \Lambda \\
(1660) & \\
\end{array}$ & -15.16 & -152.59 & -6.40 & -20.20 & 0 & -26.60 & -194.35 \\
\hline $\begin{array}{cc}\frac{1^{-}}{2}, & \Lambda \\
(1670): & \\
\frac{3}{2}^{-}, & \Lambda \\
(1690) & \end{array}$ & -43.20 & -2.22 & -2.19 & -6.32 & 0 & -8.51 & -53.93 \\
\hline $\begin{array}{cc}\frac{3}{2}, & \Sigma \\
(1660) & \\
\end{array}$ & -8.42 & -99.51 & -6.40 & -20.20 & 0 & -26.60 & -134.53 \\
\hline $\begin{array}{cc}\frac{3}{2}, & \Xi \\
(1820) & \end{array}$ & -10.80 & -49.47 & 0 & 7.18 & 0 & 7.18 & -53.09 \\
\hline
\end{tabular}

Table-4:-Energy Corrections $\left(\delta E_{B}\right)_{c m},\left(\delta E_{B}\right)_{g},\left(\delta M_{B}\right)_{\chi}$ and Physical Masses $\left(M_{B}\right)$ of $1 \mathrm{~S}, 2 \mathrm{~S}$ and $1 \mathrm{P}$ States of Light- and Strange Baryons in MeV.

\begin{tabular}{|l|c|c|c|c|c|c|c|c|}
\hline & $E_{B}^{0}$ & $\left(\delta E_{B}\right)_{c m}$ & \multicolumn{2}{|c|}{$\left(\delta E_{B}\right)_{g}$} & \multicolumn{2}{c|}{$\left(\delta M_{B}\right)_{\chi}$} & \multicolumn{2}{c|}{$M_{B}$} \\
\hline LS Muliplet & & & $\left(\delta E_{B}\right)_{g}^{m}$ & $\left(\delta E_{B}\right)_{g}^{e}$ & Total & & Cal. & Expt. \\
\hline $\mathrm{N}$ & 1068.83 & -94.75 & -110.78 & 0 & -110.78 & 76.70 & 940 & 940 \\
\hline$\Delta$ & 1068.83 & -94.75 & 110.78 & 0 & 110.78 & 147.14 & 1232 & 1232 \\
\hline$\Lambda$ & 1198.21 & -90.75 & -110.78 & 7.51 & -103.27 & 111.81 & 1116 & 1116 \\
\hline$\Sigma$ & 1198.21 & -90.75 & -92.34 & 7.51 & -84.82 & 167.64 & 1190.27 & 1193 \\
\hline$\Sigma^{*}$ & 1198.21 & -90.75 & 101.56 & 7.50 & 109.06 & 167.64 & 1384.16 & 1385 \\
\hline$\Xi$ & 1327.60 & -87.60 & -97.81 & 7.51 & -90.30 & 168.61 & 1318.31 & 1321 \\
\hline$\Xi^{*}$ & 1327.60 & -87.60 & 96.08 & 7.50 & 103.58 & 168.61 & 1512.21 & 1533 \\
\hline$\Omega^{-}$ & 1456.95 & -85.04 & 94.35 & 0 & 94.35 & 222.18 & 1688.44 & 1672 \\
\hline$\frac{1}{2}^{-}, N(1440)$ & 1798.69 & -122.12 & -8.21 & 0 & -8.21 & -228.36 & 1440.00 & 1440 \\
\hline$\frac{3}{2}^{-}, N(1520)$ & 1722.12 & -101.04 & -20.58 & 0 & -20.58 & -73.49 & 1527.01 & 1527 \\
$2^{-}$ & & & & & & & & \\
$\frac{1}{2}^{-}, N(1535)$ & & & & & & & & \\
\hline$\frac{3}{2}^{-}, \Delta(1600)$ & 1798.69 & -122.12 & 8.20 & 0 & 8.20 & -84.77 & 1600.00 & 1600 \\
\hline$\frac{1}{2}^{-}, \Delta(1620):$ & 1722.12 & -101.04 & 20.58 & 0 & 20.58 & 18.34 & 1660.00 & 1660 \\
$\frac{3}{2}^{-}, \Delta(1700)$ & & & & & & & & \\
\hline
\end{tabular}




\begin{tabular}{|l|c|c|c|c|c|c|c|c|}
\hline $\begin{array}{l}\frac{1}{2}^{-}, \Lambda(1405): \\
\frac{3}{}^{-}, \Lambda(1520)\end{array}$ & & -99.51 & -20.58 & 1.74 & -18.84 & -268.29 & 1464.00 & 1462 \\
\hline$\frac{1^{-}}{2}, \Lambda(1600)$ & 1921.94 & -120.51 & -8.21 & 1.13 & -7.08 & -194.35 & 1600.00 & 1600 \\
\hline $\begin{array}{l}\frac{1}{2}^{-}, \\
(1670):\end{array}$ & 1850.64 & -99.51 & -20.58 & 1.74 & -18.84 & -53.93 & 1678.36 & 1680 \\
$\frac{3}{2}^{-}, \Lambda(1690)$ & & & & & & & & \\
\hline$\frac{1}{2}^{-}, \Sigma(1660)$ & 1921.94 & -120.50 & -7.59 & 1.13 & -6.64 & -134.53 & 1660.45 & 1660 \\
\hline$\frac{3}{2}^{-}, \Xi(1820)$ & 1979.16 & -98.20 & -19.12 & 1.74 & -17.38 & -53.09 & 1810.47 & 1820 \\
\hline
\end{tabular}

\section{Result and conclusion:-}

In the previous chapter it has been shown that the zeroth order mass $M_{B}^{0}=E_{B}$ of ground state baryon arising out of the binding energies of the constituent quarks confined independently by a phenomenological average potential $V_{q}(r)$ which presumably represents the dominant non-perturbative multigluon interactions is subjected to certain corrections due to the residual quark gluon interaction and GBE interaction between the constituent quarks, together with that due to the spurious center-of-mass motion. All of these corrections can be treated independently, as though they are of the same order of magnitude, so that the physical mass of baryon can be obtained as

$$
M_{B}=E_{B}^{0}+\left(\Delta E_{B}\right)_{c m}+\left(\Delta E_{B}\right)_{\chi}+\left(\Delta E_{B}\right)_{g}^{m}+\left(\Delta E_{B}\right)_{g}^{e}
$$

where $\left(\Delta E_{B}\right)_{c m}$ is the c.m correction to baryon mass (eqn. 2.28), $\left(\Delta E_{B}\right)_{\chi}$ with $\chi=(\pi, \eta, \mathrm{k})$ is the GBE correction (eqn. 2.37), and $\left[\left(\Delta E_{B}\right)_{g}^{m}+\left(\Delta E_{B}\right)_{g}^{e}\right]$ is the colour magnetic and electric interaction energies arising out of OGE interactions [eqn. (2.41) and eqn.(2.42)].

The quantitative evaluation of the zeroth order energy $E_{B}^{0}$ of the baryon core, the c.m correction $\left(\Delta E_{B}\right)_{c m}$, the GBE correction $\left(\Delta E_{B}\right)_{\chi}$ and OGE correction $\left(\Delta E_{B}\right)$ with in the frame-work of the model primarily involves the potential parameters $\left(\mathrm{a}, V_{0}\right)$, the quark masses $m_{q}$ and the corresponding binding energy $E_{n l}^{q}$ along with relevant model quantities. In the present model, The quark masses $m_{q}$ and the potential parameter 'a' are suitable chosen and different values of the potential parameter " $V_{0}^{n l}$ " are appropriately fixed for $1 \mathrm{~s}, 2 \mathrm{~s}$, and $1 \mathrm{p}$ states of baryons so as to obtain a reasonable fit to the baryon resonance spectra taking into account the energy corrections due to GBE and OGE interactions between the constituent quarks together with that due to c.m motion. Here, we choose the quark masses $m_{q}$ and the potential parameter 'a' as $\left(m_{u}=m_{d}, m_{s}\right)=(278,447) \mathrm{Mev}$ and 'a' $=200 \mathrm{Mev}$ and fix the values of the parameters $V_{0}^{n l}$

$$
\left(V_{0}^{n l}, V_{0}^{n l}, V_{0}^{n l}\right)=(-182,-125,-74) M e V
$$

For $1 \mathrm{~s}, 2 \mathrm{~s}, \& 1 \mathrm{p}$ baryon states respectively. With these values of the parameters the solution of the energy eigen values of eqn (2.5) yields the individual quark binding energies $E_{n l}^{q}$ for $1 \mathrm{~s}, 2 \mathrm{~s}$, and $1 \mathrm{p}$ states respectively as

$$
\begin{gathered}
\left(E_{1 s}^{u}=E_{1 s}^{d}, E_{1 s}^{s}\right)=(346,286.3) \mathrm{MeV} \\
\left(E_{2 s}^{u}=E_{2 s}^{d}, E_{2 s}^{s}\right)=(598,713.62) \mathrm{MeV} \\
\left(E_{1 p}^{u}=E_{2 p}^{d}, E_{2 s}^{s}\right)=(574,692.3) \mathrm{MeV}
\end{gathered}
$$

We then evaluate with the help of a standard numerical method and calculate the term $T_{i j}^{e m}$ from (2.41) and (2.42) which are necessary for computing the corrections due to OGE and GBE interactions respectively. These expressions are calculated for $1 \mathrm{~s}, 2 \mathrm{~s}$ and $1 \mathrm{p}$ states and are displaced in Table ( 2 ).

Indeed, in the chiral limit there is only one coupling constant for all Goldstone bosons. Due to explicit chiral symmetry breaking the coupling constant for $\pi, \eta$ and $\mathrm{k}$ may become different. 
However, in order to prevent a proliferation of the free parameters we try to keep the number of free parameters as small as possible and assume a single phenomenological pion coupling constant $f_{N N \pi}=0.283$ for all mesons $(\pi, \eta$, $\mathrm{k}$ ) and this value is used here to compute GBE corrections. The calculated results for the contribution from the GBE interaction to baryon resonance spectra are presented in Table (3). The energy corrections and the results obtained for the baryon resonance spectra are displaced in Table (4 ). The calculated values of baryon resonance spectra are found to agree reasonably well with the experiment. It is found that the OGE corrections require a value of quarkgluon coupling constant $\alpha_{c}=0.50$ which is consistent with the idea of treating OGE effects in low order perturbation theory. Extension of this work to the study the mass spectra for other four ${ }^{*}$ and three star ${ }^{*}$ resonances of the particle data group.
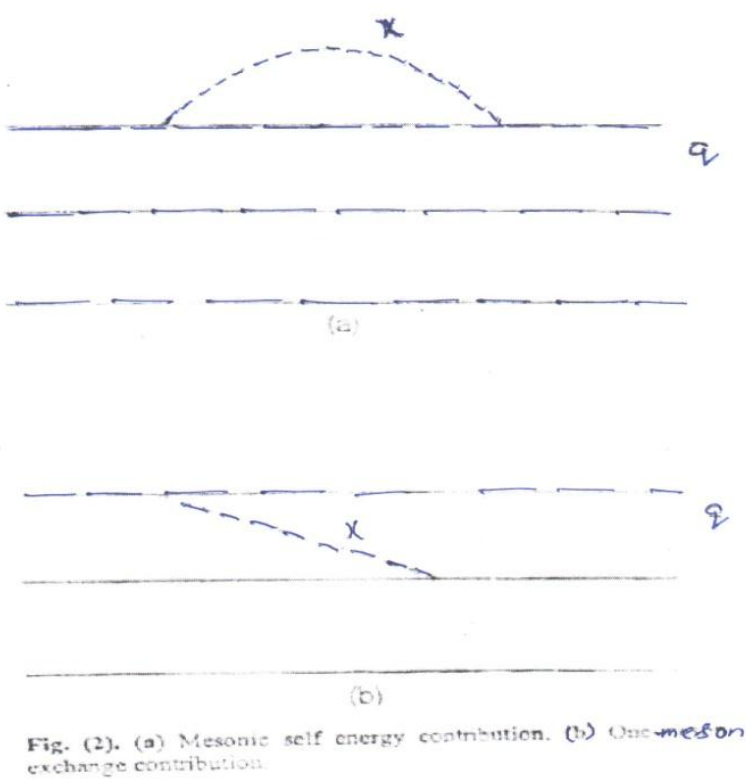

\section{Acknowledgements:-}

The author is grateful to Prof. S. N. Jena, Retired Professor of Physics, P.G. Department of Physics, Berhampur University, Berhampur, Odisha, India for his valuable suggestions and useful discussions on this work.

\section{References:-}

1. S. N. Jena, M. R. Behera and S. Panda, Pramana- J. Phys. 5,711(1998).

2. S. N. Jena, M. R. Behera and S. Panda, Phys. Rev. D55,291(1997).

3. C. Amsler, M. Dosor and M. Antorelli, "the particle data group" the reviewed of particle physics, Phys. Lett. B667,1-6(2008).

4. L. Ya. Glozman, W. Plesses, K. Varga, R. F. Wagenbrun, Phys. Rev. D58,094030(1998).

5. L. Ya. Glozman and D. O. Riska, Phys. Rep, 268,263(1996).

6. L. Ya. Glozman, Nucl. Phys. A663,103(2000).

7. S. Weinberg, Physica A96,327(1979). Manohar and H. Geogi, Phys. B234,189(1984).

8. D. I. Diaknov and V. Yu. Petrov, Nucl. Phys. B272,457(1986).

9. M. Gellmann and M. Levy, Nuovo Cimento 16,705.

10. S. P. Klevansky, Rev. Mod. Phys.64,649(1992).

11. J. F. Donoshue and K. Johnson, Phys. D21,1975(1980).

12. C. W. Wing, Phys. Rev. 15C,283(1975); J. A. Bartelski, A. Szymaeha, L. Ankcewicz and S. Tafar.

13. C. W. Wong,Phys. Rev. D24,1416(1981).

14. S. Aoki etal. Phys.Rev. Lett.82,4392(1999).

15. S. Weinberg, Physica A96,327(1979); A. Manohor and H. Georgi,Nucl.Phys B234,189(1984).

16. L. Ya. Glozman, Z. Papp, W. Plessua, K. Varga and R. F. Wagenbrunn, Phys.Rev.C57. 